\title{
Semiclassical Quantization of Spinning Quasiparticles in Ballistic Josephson Junctions
}

\author{
François Konschelle, ${ }^{1}$ F. Sebastián Bergeret, ${ }^{1,2}$ and Ilya V. Tokatly ${ }^{3,4}$ \\ ${ }^{1}$ Centro de Física de Materiales (CFM-MPC), Centro Mixto CSIC-UPV/EHU, Manuel de Lardizabal 5, E-20018 San Sebastián, Spain \\ ${ }^{2}$ Donostia International Physics Center (DIPC), Manuel de Lardizabal 4, E-20018 San Sebastián, Spain \\ ${ }^{3}$ Nano-Bio Spectroscopy group, Departamento Física de Materiales, Universidad del País Vasco, Avenida Tolosa 72, \\ E-20018 San Sebastián, Spain \\ ${ }^{4}$ IKERBASQUE, Basque Foundation for Science, E-48011 Bilbao, Spain
}

(Received 12 January 2016; published 8 June 2016)

\begin{abstract}
A Josephson junction made of a generic magnetic material sandwiched between two conventional superconductors is studied in the ballistic semiclassic limit. The spectrum of Andreev bound states is obtained from the single valuedness of a particle-hole spinor over closed orbits generated by electron-hole reflections at the interfaces between superconducting and normal materials. The semiclassical quantization condition is shown to depend only on the angle mismatch between initial and final spin directions along such closed trajectories. For the demonstration, an Andreev-Wilson loop in the composite positionparticle-hole-spin space is constructed and shown to depend on only two parameters, namely, a magnetic phase shift and a local precession axis for the spin. The details of the Andreev-Wilson loop can be extracted via measuring the spin-resolved density of states. A Josephson junction can thus be viewed as an analog computer of closed-path-ordered exponentials.
\end{abstract}

DOI: 10.1103/PhysRevLett.116.237002

In the past years, the spin-orbit and spin-splitting effects in superconducting heterostructures $[1,2]$ have received a great deal of attention in the context of an emerging superconducting spintronics $[3,4]$ and in connection with possible realizations of Majorana bound states in nanowires [5]. A Josephson junction with a magnetoactive normal bridge exemplifies a prototype structure hosting such a kind of spin interactions. The physics of superconductor-normalmetal-superconductor $(S / N / S)$ ballistic Josephson junctions is mainly determined by the so-called Andreev bound states (ABSs) localized in the $N$ region. These states, which carry a significant fraction of the Josephson supercurrent $[6,7]$, have been extensively studied in ballistic superconducting point contacts [8-10].

Theoretically, the quantization of states trapped in some classically allowed region can be understood from the BohrSommerfeld quantization rule $[11,12]$, which requires the phase accumulated along a closed classical trajectory to be a multiple of $2 \pi$. In a ballistic $S / N / S$ junction, the trapping in the $N$ region occurs due to Andreev reflections with the conversion of the incident electron to the reflected hole and vice versa at the $S / N$ interfaces [13]. Each Andreev reflection brings a phase shift $\theta(E)=\arccos (E / \Delta)$, where $E<\Delta$ is the energy measured with respect to the Fermi level [14]. The classical loop trajectory is now defined in the space composed of the position and particle-hole subspaces. In the position subspace, the electron and the reflected hole accumulate the phase equal to $2 E L / v$, where $L$ is the distance between the $S$ electrodes and $v$ is the component of the velocity perpendicular to the junction plane. From the two Andreev reflections (shifts in the particle-hole subspace), the phase acquires the contribution $2 \theta(E) \pm \varphi$, depending on the propagating direction, where $\varphi$ is the phase difference between the two $S$ electrodes (see Fig. 1) [16]. Hence, the quantization condition for ABSs reads $2 E_{n} L /|v|-2 \theta\left(E_{n}\right)+\operatorname{sgn}(v) \varphi=2 n \pi$. The spin-orbit

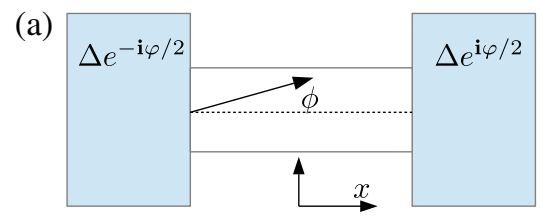

(b)
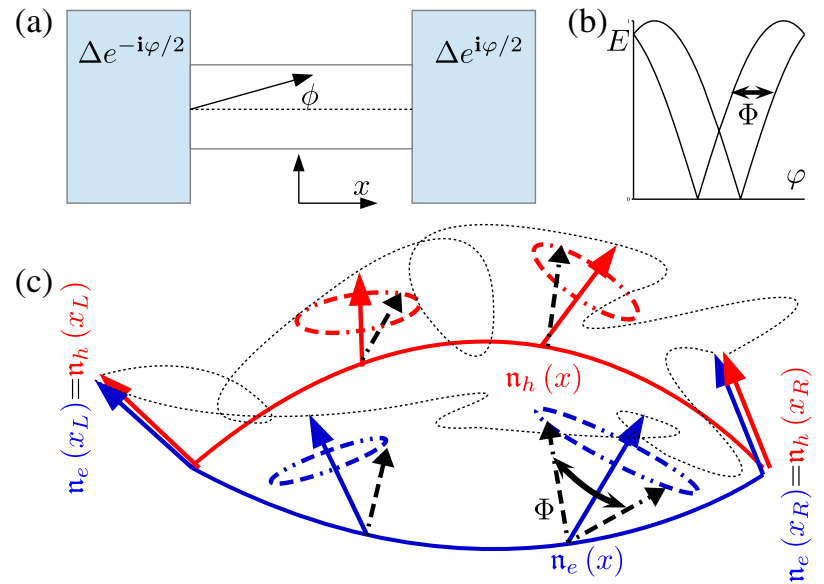

FIG. 1. (a) Sketch of the Josephson junction, with $\phi$ the Fermi angle and $\varphi$ the phase difference. (b) Sketch of the energy of the Andreev bound states, versus $\varphi$, spin-split by $\Phi$. (c) The spin structure of the Andreev-Wilson loop that starts at point $x$. The local $\mathbf{n}_{e}$ (blue) vector evolves from the left to the right interfaces, where it is equal to $\mathbf{n}_{h}$ (red) which propagates in the opposite direction $\left[\mathbf{n}_{e}\left(x_{L, R}\right)=\mathbf{n}_{h}\left(x_{L, R}\right)\right]$. The spin direction (black dotted arrows) precesses around the local vectors $\mathbf{n}_{e, h}$ at a constant latitude (red and blue dotted projective circles). After completing the loop, the spin rotates an angle $\Phi$ between the initial and final states. This angle determines the phase shift between the two spin-split Andreev bound states [see (b)]. 
coupling (SOC) and spin-splitting (exchange or Zeeman), possibly textured fields in a magnetic material, generate precession of the electron and hole spins, which should modify the properties of ABSs. How the semiclassical condition is modified in the presence of generic spindependent fields is an open question we address in this Letter.

We identify an additional phase shift $\Phi$ originating from the spin precession generated by an effective magnetic field in the $N$ region; see Eq. (8). This precession preserves, as in the nonsuperconducting case $[17,18]$, the latitude with respect to the local spin quantization axis $\mathbf{n}$, which obeys a classical equation [Eq. (7)]. We first derive the modified quantization condition, determine the subgap spectrum of a ballistic $S / N / S$ junction, and finally demonstrate by solving the quasiclassical Eilenberger equation how $\Phi$ and $\boldsymbol{n}$ enter the expressions of other physical quantities like the Josephson current or the spin-resolved local density of states. Our results generalize the quasiclassical theory of spinning electrons described by Dirac and/or Pauli equations $[17,18]$ to the case of quasiclassical motion of Bogoliubov quasiparticles in superconducting structures. In the normal state, a nonadiabatic spin precession of electrons moving along cyclotron orbits is revealed experimentally in anomalous Shubnikov-de Haas oscillations of magnetoresistance [19]. Here we demonstrate that all details of highly nontrivial spin dynamics of bogolons forming $\mathrm{ABSs}$ can be extracted from the observable properties of Josephson junctions.

We consider the semiclassical Bogoliubov-de Gennes $(\mathrm{BdG})$ bispinor wave function $(u, v)=e^{\mathbf{i k} \cdot \mathbf{r}}(\psi, \chi)$, where $|\mathbf{k}|=k_{F}$ is the Fermi momentum and the electron $\psi$ and hole $\chi$ spinors are slowly varying on the scale of $k_{F}^{-1}$ [20]. The behavior of the wave function in the presence of an effective, coordinate- and velocity-dependent, magnetic field $\boldsymbol{B}(\boldsymbol{v}, x)$, which couples to the electron and hole spins and describes generic SOC and exchange or Zeeman spin splitting, is governed by the following BdG equations:

$$
\begin{aligned}
-\mathbf{i} \boldsymbol{v} \cdot \boldsymbol{\nabla} \psi+\boldsymbol{B}(\boldsymbol{v}, x) \cdot \boldsymbol{\sigma} \psi+\Delta \chi & =E \psi, \\
\mathbf{i} \boldsymbol{v} \cdot \boldsymbol{\nabla} \chi+\boldsymbol{B}(-\boldsymbol{v}, x) \cdot \boldsymbol{\sigma} \chi+\Delta^{*} \psi & =E \chi .
\end{aligned}
$$

We do not impose any restriction on the $\boldsymbol{v}$ or $x$ dependence of $\boldsymbol{B}$ that, in principle, may correspond to any magnetic texture and any type of SOC.

After being transported over a closed Andreev trajectory that starts at $x$ within the $N$ region $\left(x_{L}<x<x_{R}, \Delta=0\right)$, the BdG bispinor should return to itself:

$$
\left(\begin{array}{c}
\psi(x) \\
\chi(x)
\end{array}\right)=e^{\mathrm{i}[2 E L / v-2 \operatorname{sgn}(v) \theta(E)+\varphi]}\left(\begin{array}{c}
W_{e} \psi(x) \\
W_{h} \chi(x)
\end{array}\right),
$$

where $v=v_{F} \cos \phi$ and $\phi$ is the angle between the semiclassical trajectory and the junction axis $x$. In Eq. (2), the effect of spin-dependent fields is encoded in the electron and hole spin rotation operators

$$
\begin{aligned}
& W_{e}(x)=U\left(x, x_{L}\right) \bar{U}\left(x_{L}, x_{R}\right) U\left(x_{R}, x\right), \\
& W_{h}(x)=\bar{U}\left(x, x_{R}\right) U\left(x_{R}, x_{L}\right) \bar{U}\left(x_{L}, x\right),
\end{aligned}
$$

which are defined via the path-ordered spin propagator

$$
U\left(x_{2}, x_{1}\right)=P \exp \left\{-\frac{\mathbf{i}}{v} \int_{x_{1}}^{x_{2}} \boldsymbol{B}(v, x) \cdot \boldsymbol{\sigma} d x\right\}
$$

and its time-reversal conjugate $\bar{U}$ [21].

The $W_{e, h}$ operators, which transport spinors over a closed trajectory, are reminiscent of the Wilson loop operators in the SU(2) gauge theory. They take into account the electron-hole conversions at the $S / N$ interfaces, and they are thus defined along the Andreev loop. For this reason, we call $W_{e, h}$ the Andreev-Wilson (AW) loop operators, which describe transport along a loop in the composite position $\otimes$ particle-hole space [22].

Several properties of $W_{e, h}$ are discussed in Supplemental Material [24]. The most remarkable is that for any $\boldsymbol{B}(v, x)$ the trace of $W_{e, h}(x)$ is $x$ independent; i.e., it does not depend on the initial point of the loop. Hence, $W_{e, h}(x)$ can be parametrized by local unit vectors $\mathfrak{n}_{e, h}(x)$ and a coordinate independent angle $\Phi$ :

$$
W_{e, h}(x)=\exp \left\{\mathbf{i}\left[\mathbf{n}_{e, h}(x) \cdot \boldsymbol{\sigma}\right] \Phi\right\} .
$$

The vectors $\mathfrak{n}_{e, h}(x)$ satisfy the classical equation of a magnetic moment precessing in a magnetic field (see [24]):

$$
\pm v \partial_{x} \boldsymbol{n}_{e, h}=2 \boldsymbol{B}( \pm v, x) \times \boldsymbol{n}_{e, h} .
$$

Since Andreev reflections preserve the spin, one has the boundary condition $\mathfrak{n}_{e}\left(x_{R, L}\right)=\mathfrak{n}_{h}\left(x_{R, L}\right)$ [see (3) and (4)] uniquely defining $\boldsymbol{n}_{e, h}(x)$. One can easily see that the expectation values of the electron and hole spins, $\mathbf{s}_{e}(x)=$ $\psi^{\dagger} \boldsymbol{\sigma} \psi$ and $\mathbf{s}_{h}(x)=\chi^{\dagger} \boldsymbol{\sigma} \chi$, have $x$-independent projections on the local directions $\mathbf{n}_{e}(x)$ and $\mathbf{n}_{h}(x)$, respectively [24]. Notice that $\boldsymbol{n}_{e . h}$ and $\Phi$ depends on $v$. From Eqs. (3) and (4), one can easily check that $\left.\mathbf{n}_{e} \cdot \Phi\right|_{v}=-\left.\mathbf{n}_{h} \cdot \Phi\right|_{-v}$. Based on the electron-hole symmetry, we impose that $\boldsymbol{n}_{e}(v)=\mathbf{n}_{h}(-v)$. From this follows that $\Phi(v)=-\Phi(-v)$.

It is now possible to give a semiclassical interpretation of the AW loops (see Fig. 1) inspired by the picture of quantization for spinning particles proposed in Refs. [17,18]. When transported along the loop, the "classical spins" $\mathbf{s}_{e, h}(x)$ of electrons and holes precess around local axes, $\boldsymbol{n}_{e}(x)$ for the electrons and $\boldsymbol{n}_{h}(x)$ for the holes, in such a way that latitude with respect to those axes is always preserved. If, for example, one starts the AW loop with a right-moving electron at position $x$, the electron spin will precess around the local $\mathfrak{n}_{e}$ until it reaches the right electrode. At this point, the electron is reflected as a hole. The resulting hole propagates from the right to the left interface with spin precessing around the local holelike 
axis $\mathfrak{n}_{h}$. At the left interface, the inverse process takes place, and the AW loop ends up with an electron precessing around the $\mathbf{n}_{e}(x)$ axis again. While the rotation axis $\boldsymbol{n}_{e, h}$ after completing the loop is preserved, the spin itself does not return to its original direction. There is an angle mismatch $\Phi$, at fixed latitude with respect to $\mathbf{n}_{e}(x)$, between the initial and final spin. Being position independent [since $2 \cos \Phi=\operatorname{Tr}\left\{W_{e, h}\right\}$; see (3)-(6)], this angle mismatch has a global meaning: It corresponds to the phase acquired by the wave function after one turn. The single valuedness of the wave function after a complete period, expressed by Eq. (2), leads to the generalized semiclassical quantization condition:

$$
\frac{2 E_{n, s} L}{|v|}-2 \arccos \frac{E_{n, s}}{\Delta}+\operatorname{sgn}(v)[\varphi+s \Phi]=2 n \pi,
$$

which determines the spectrum of ABSs, with $s= \pm 1$ being the spin projection. The appearance of finite $\Phi$ lifts the spin degeneracy of the ABS. From Eqs. (3)-(5), one can verify that the spin splitting occurs only if the effective magnetic field $\boldsymbol{B}$ breaks the time-reversal symmetry; otherwise, the AW-loop operators are trivial [25].

As an example, we consider the widely studied $S / F / S$ junction ( $F$ is a ferromagnet) [1,2]. When the exchange field points along the $z$ axis, $\mathbf{m}_{e}=\mathbf{n}_{h}=\hat{z}$ are constant in space and $\Phi=2 \int_{x_{L}}^{x_{R}} h_{z}(x) d x / v$. In particular, this reduces to the usual $\Phi=2 h L / v$ for a monodomain $S / F / S$ [28,29] and zero $\Phi$ for an oscillating exchange field with opposite domains of equal length [30]. In fact, the previously known results for various specific $S / F / S$ junctions follow immediately from our general formulation that is valid for an arbitrary exchange field and SOC. The main message here is that all the spin-related features are encoded in the phase $\Phi$ and local unit vectors $\boldsymbol{n}_{e, h}(x)$.

Clearly, the knowledge of the subgap spectral properties is not sufficient to fully characterize the physics of the $S / N / S$ junction. To understand, for example, how the Josephson current is affected by the spin-dependent interactions and finite temperature, or whether a finite magnetic moment can be created in the junction, one needs to extend the formalism and take into account all energies in the spectrum and the electronic distribution functions. For this, we introduce the quasiclassical Green's function, solve the Eilenberger equation for the $S / N / S$ junction, and explore how the vectors $\mathbf{n}_{e, h}$ and the phase $\Phi$, associated with the AW loop, manifest in physical observables.

For a clean $S / N / S$ junction in the presence of an effective magnetic field $\boldsymbol{B}=\boldsymbol{h}+\boldsymbol{b}(v)$, where $\boldsymbol{h}$ is the spin-splitting or Zeeman field and $\boldsymbol{b}(-v)=-\boldsymbol{b}(v)$ describes the SOC, the Eilenberger equation reads [31,32]

$$
\mathbf{i} v \partial_{x} \check{g}=\left[\tau_{3} E+\left(\tau_{3} \boldsymbol{h}+\boldsymbol{b}\right) \cdot \boldsymbol{\sigma}+\check{\Delta}, \check{g}(x)\right] .
$$

Here $\check{g}$ is the matrix Green's function in the Nambu and spin space, and $\check{\Delta}=\Delta e^{\mathbf{i} \tau_{3} \varphi / 2} \mathbf{i} \tau_{2} e^{-\mathbf{i} \tau_{3} \varphi / 2}$ is a $4 \times 4$ matrix proportional to the identity matrix in spin space and the Pauli matrices $\tau_{i}$ spanning the Nambu space. We assume that $\Delta$ is constant and nonzero in $S$ electrodes only, whereas $\boldsymbol{B}$ is present in the $N$ region. In Eq. (9), we keep only terms in the lowest order in $\left(\xi p_{F}\right)^{-1}$, where $\xi$ is any characteristic length scale $\xi$ involved in the problem. Higher-order terms are responsible for the appearance of an anomalous phase in $S F S$ structures and an additional source for singlet-triplet conversion $[27,33]$.

By assuming continuity of the Green's functions across the interfaces, we obtain for the electron component $g$ of the Green's function in $N$ [the $(1,1)$ component of $\check{g}$ in Nambu space] [34]:

$$
g(x, E)=-\mathbf{i} \sum_{s= \pm} \frac{1}{2}\left[1+s \mathbf{n}_{e}(x) \cdot \boldsymbol{\sigma}\right] T_{s}(E),
$$

with $s$ the spin projection and

$$
T_{s}(E)=\tan \left(\frac{E L}{|v|}+\arcsin \frac{E}{\Delta}+\operatorname{sgn}(v)\left[\frac{\varphi}{2}+s \frac{\Phi}{2}\right]\right) .
$$

The poles of $T_{s}$ for energies $E \leq \Delta$ represent the ABSs, and we thus recover Eq. (8) explicitly. It is remarkable that the precession angle mismatch $\Phi$ and the local spin precession axis $\mathbf{n}_{e}(x)$ obtained from our previous semiclassical consideration enter explicitly the Green's function. We also note that factors $\frac{1}{2}\left[1 \pm \mathbf{n}_{e}(x) \cdot \boldsymbol{\sigma}\right]=\left|\psi_{ \pm}(x)\right\rangle\left\langle\psi_{ \pm}(x)\right|$ in Eq. (10) are exactly the projectors on the states with spin up or down with respect to the local direction $\mathbf{n}_{e}(x)$.

The quasiclassical Green's function (10) determines physical observables like the density of states, given by

$$
\frac{N(E)}{N_{0}}=\frac{1}{\pi} \lim _{\epsilon \rightarrow 0} \Re \sum_{s= \pm}\left\langle T_{s}(E+\mathbf{i} \epsilon)\right\rangle
$$

and the charge current through the junction $j=(1 / 4) \mathbf{i} \pi e N_{0} k_{B} T \sum_{\omega_{n}} \operatorname{Tr}\langle v g\rangle$, where $\langle\ldots\rangle$ denotes averaging over the Fermi surface and the sum is over the Matsubara frequencies $\omega_{n}=2 \pi k_{B} T(n+1 / 2)$. After the substitution of Eq. (10), the charge current has the form

$$
j=e \frac{\pi}{4} N_{0} k_{B} T \sum_{\omega_{n}} \sum_{s= \pm}\left\langle v T_{s}\left(\mathbf{i} \omega_{n}\right)\right\rangle .
$$

This expression is valid for any Fermi surface, length of the junction, magnetic interaction, and temperature. In that respect, it generalizes previous results obtained in ballistic $S / F / S$ systems $[1,2,35,36]$ to an arbitrary spin texture. Equation (13) shows that the current phase relation depends only on the parameter $\Phi$ irrespective of its origin. For example, when $T$ is close to the critical temperature $T_{c}$, the above expression simplifies to 


$$
\lim _{T \approx T_{c}} j=\frac{2 e N_{0} \Delta^{2}}{\pi^{2} T_{c}}\left\langle|v| e^{-2 \pi L T_{c} /|v|} \cos \Phi\right\rangle \sin \varphi,
$$

which contains only the global magnetic phase shift $\Phi$, appearing as a modulation of the Josephson current-phase relation.

It is clear from Eqs. (12) and (13) that spin-independent observables, such as the total density of states or the charge current, do not depend on $\mathbf{n}_{e}$ and hence are constant in the $N$ region. In order to obtain information about the vector $\mathbf{n}_{e}$, one needs to measure spin-dependent observables. We introduce the spectral spin density polarized in the $a$ direction (spin-resolved density of states) defined by

$$
\frac{N^{a}(E)}{N_{0}}=\lim _{\epsilon \rightarrow 0} \frac{\Re\left\langle\operatorname{Tr}\left\{\sigma^{a} g\right\}\right\rangle}{\pi}=\frac{1}{\pi} \Re \sum_{s= \pm}\left\langle s \mathbf{n}_{e}^{a} T_{s}\right\rangle
$$

that can be determined by means of tunneling spectroscopy similar to the ABS spectroscopy done in carbon nanotubes connected to superconductors [37]. If instead of nanotubes one uses semiconducting wires with a strong enough intrinsic SOC, a Zeeman field will induce a finite $\Phi$ lifting the degeneracy of the ABS. The phase $\Phi$ will also manifest itself through the Josephson current according to Eq. (14). In a similar experimental setup, one can have access to the spectral spin density $N^{a}(15)$. Suppose the detector is fully polarized (i.e., in a half-metallic limit) and magnetized along the $a$ direction; by performing two measurements of the differential conductance for opposite magnetizations of the tunneling probe, $G_{a}$ and $G_{-a}$, one determines $G_{a}(V)-G_{-a}(V) \propto \mathfrak{n}^{a} \sum_{s= \pm 1} s T_{s}(V)$. Thus, by measuring the total and spin-dependent density of states, one can have an access to the parameters $\Phi$ and $\boldsymbol{n}_{e}$ which determine the full AW loop operator (3).

Previous results have been obtained assuming a perfect contact between the $S$ electrodes and the $N$ link. One can, however, generalize the Bohr-Sommerfeld quantization condition when adding scattering interfaces between the $S$ and $N$ materials [38]. Assuming the left $(L)$ and right $(R)$ interfaces with transmission probabilities $T_{L, R}=1-R_{L, R}$ and reflection coefficients $r_{L, R}$ with $\left|r_{L, R}\right|^{2}=R_{L, R}$, one obtains for a single channel junction (see [24])

$$
\begin{aligned}
& \cos \left(\frac{2 E L}{v_{F}}+s \Phi+2 \theta\right)-\left(R_{R}+R_{L}\right) \cos \left(\frac{2 E L}{v_{F}}+s \Phi\right) \\
& \quad+R_{R} R_{L} \cos \left(\frac{2 E L}{v_{F}}+s \Phi-2 \theta\right) \\
& \quad=T_{L} T_{R} \cos \varphi-2\left(r_{L} r_{R}+r_{L}^{*} r_{R}^{*}\right) \sin ^{2} \theta
\end{aligned}
$$

for the condition of existence of ABSs. Equation (16) generalizes results known for the case of $S / N / S$ systems without magnetic interactions; the case $\Phi=0, r_{L}=0$ has been obtained in Ref. [39]. Supposing a strong enough magnetic texture, such that $\Phi \gg 2 E L / v_{F}$, one can plot the
ABS of a short Josephson junction for different junction transparency. As an example, we consider a symmetric junction with $\delta$ barriers at the interfaces: $r_{L}=r_{R}=$ $-\mathbf{i} Z /(1+\mathbf{i} Z)$; see Fig. 2 . In contrast to the case $\Phi=0$, where any finite barrier strength $(Z \neq 0)$ opens a gap, a finite $\Phi$ leads to a critical value of $Z$ below which zeroenergy states exists. As one can infer by comparing the upper and lower rows in Fig. 2, this critical value of $Z$ increases by increasing $\Phi$.

In conclusion, we have derived the semiclassical quantization condition for a $S / N / S$ Josephson junction when the normal region exhibits a generic SOC and exchange or Zeeman field. We obtained the spectrum of ABS Eq. (8) and the quasiclassical Green's function Eq. (10) and analyzed several physical observables in the presence of such a generic spin-dependent field. We demonstrated that all the properties of the junction are expressed in terms of two fundamental parameters: $\Phi$ and $\boldsymbol{n}(x)$; see Fig. 1. These two parameters have a clear semiclassical meaning. The unit vector $\mathbf{m}(x)$ describes the local spin quantization axis about which a classical spin precesses at a constant latitude while propagating through the junction. The magnetic phase $\Phi$ corresponds to the mismatch of the precession angles after a quasiparticle completes the closed Andreev orbit. $\Phi$ enters explicitly the expression for the Josephson current, while $\mathfrak{n}$ can be accessed experimentally by measuring the spin-resolved density of states. A magnetic Josephson junction can thus be used as an analog computer of path-ordered loop operators (3)-(6).

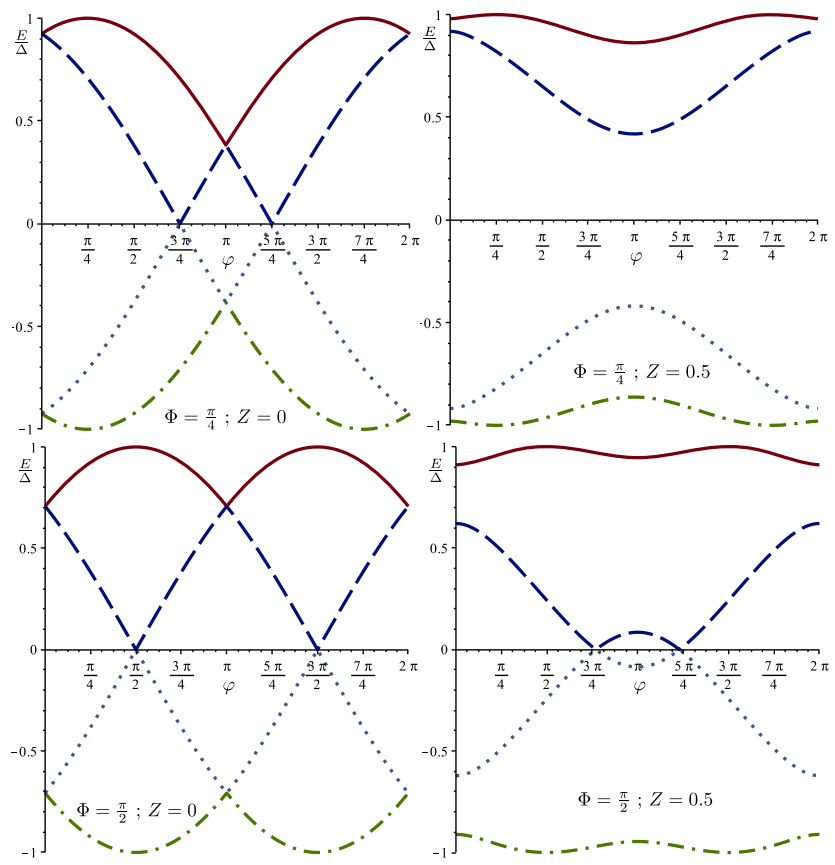

FIG. 2. The spin-split ABS energy $E / \Delta$ in a $S / N / S$ junction with a finite interface transparency versus the phase difference $\varphi$, in the limit $\Phi \gg 2 E L / v_{F}$ from Eq. (16). Left column: The strength of the interface $\delta$ barrier $Z=0$; right column: $Z=0.5$. 
We thank D. Bercioux and V. N. Golovach for stimulating remarks. Discussions with $\mathrm{H}$. Bouchiat, P. Joyez, H. Pothier, and L. Tosi during the annual meeting of the GDRI de Physique Mésoscopique, Aussois, were particularly appreciated. The work of F. S. B. and F. K. was supported by Spanish Ministerio de Economía y Competitividad (MINECO) through Project No. FIS2014-55987-P and the Basque Government under UPV/EHU Project No. IT-756-13. I. V. T. acknowledges support from the Spanish Grant No. FIS2013-46159-C3-1-P and from the "Grupos Consolidados UPV/EHU del Gobierno Vasco" (Grant No. IT578-13).

[1] A. I. Buzdin, Rev. Mod. Phys. 77, 935 (2005).

[2] F. S. Bergeret, A. F. Volkov, and K. B. Efetov, Rev. Mod. Phys. 77, 1321 (2005).

[3] M. Eschrig, Phys. Today 64, No. 1, 43 (2011).

[4] J. Linder and J. W. A. Robinson, Nat. Phys. 11, 307 (2015).

[5] M. Franz, Nat. Nanotechnol. 8, 149 (2013).

[6] C. Beenakker, Rev. Mod. Phys. 69, 731 (1997).

[7] A. Furusaki and M. Tsukada, Solid State Commun. 78, 299 (1991).

[8] M. L. Della Rocca, M. Chauvin, B. Huard, H. Pothier, D. Esteve, and C. Urbina, Phys. Rev. Lett. 99, 127005 (2007).

[9] L. Bretheau, Ç. Ö. Girit, H. Pothier, D. Esteve, and C. Urbina, Nature (London) 499, 312 (2013).

[10] C. Janvier, L. Tosi, L. Bretheau, C. O. Girit, M. Stern, P. Bertet, P. Joyez, D. Vion, D. Esteve, M. F. Goffman, H. Pothier, and C. Urbina, Science 349, 1199 (2015).

[11] A. A. Abrikosov, Fundamentals of the Theory of Metals (North-Holland, Amsterdam, 1988).

[12] A. Messiah, Mécanique Quantique (Dunod, Paris, 1995) (French reprint of the 1958 edition).

[13] A. F. Andreev, Sov. Phys. JETP 19, 1228 (1964).

[14] In the strict semiclassical limit, i.e., $E \rightarrow 0, \theta(0)=\pi / 2$ is the usual Maslov index at a turning point [15].

[15] K. P. Duncan and B. L. Györffy, Ann. Phys. (Amsterdam) 298, 273 (2002).

[16] C. W. J. Beenakker and H. van Houten, Phys. Rev. Lett. 66, 3056 (1991).

[17] S. Keppeler, Phys. Rev. Lett. 89, 210405 (2002).

[18] S. Keppeler, Ann. Phys. (Amsterdam) 304, 40 (2003).

[19] S. Keppeler and R. Winkler, Phys. Rev. Lett. 88, 046401 (2002).

[20] We assume the validity of the semiclassical approach. This means that we assume that all lengths involved in the problem are larger than the Fermi wavelength and all energies, in particular, the SOC and Zeeman splitting, are smaller than the Fermi energy.
[21] Time-reversal conjugation of an operator $O\left(\mathbf{p}_{\mathbf{F}}\right)$ is given by $\bar{O}\left(\mathbf{p}_{\mathbf{F}}\right)=\sigma^{y} O^{*}\left(-\mathbf{p}_{\mathbf{F}}\right) \sigma^{y}$

[22] In the non-Abelian gauge theory, a path-ordered exponential describing a parallel transport of a spinor field over a closed loop in the real space is called a Wilson operator, and its trace is called a Wilson loop [23]. Equations (3) and (4) also represent the transport along a loop, now generated by the Andreev reflections, and thus closes only in the combined position $\otimes$ particle-hole space. To recall this subtlety, we call $W_{e, h}$ the Andreev-Wilson (AW) loop operators.

[23] M. E. Peskin and D. V. Schroeder, An Introduction to Quantum Field Theory (Westview Press, Boulder, CO, 1995).

[24] See Supplemental Material at http://link.aps.org/ supplemental/10.1103/PhysRevLett.116.237002 for a statement of the mathematical conventions followed in this study, the mathematical structure behind Fig. 1, a lengthy discussion of the detection of the Andreev-Wilson loop, and the derivation of Eq. (16).

[25] The fact that only a nonzero Zeeman field leads to the spin splitting of the ABSs is a consequence of the leading-order semiclassical approximation. Beyond this approximation, a lifting of the spin degeneracy can be achieved by SOC only (see, e.g., [26]) if the phase difference $\varphi$ is nonzero. This is directly linked to the appearance of a finite magnetic moment by passing a current through weak link with a SOC [27].

[26] O. V. Dimitrova and M. V. Feigel'man, J. Exp. Theor. Phys. 102, 652 (2006).

[27] F. Konschelle, I. V. Tokatly, and F. S. Bergeret, Phys. Rev. B 92, 125443 (2015).

[28] L. Bulaevskii, A. I. Buzdin, and S. Panjukov, Solid State Commun. 44, 539 (1982).

[29] A. I. Buzdin, L. Bulaevskii, and S. V. Panyukov, Sov. Phys. JETP 35, 178 (1982).

[30] Y. M. Blanter and F. W. J. Hekking, Phys. Rev. B 69, 024525 (2004).

[31] F. S. Bergeret and I. V. Tokatly, Phys. Rev. B 89, 134517 (2014).

[32] F. Konschelle, Eur. Phys. J. B 87, 119 (2014).

[33] C. R. Reeg and D. L. Maslov, Phys. Rev. B 92, 134512 (2015).

[34] Details of the derivation will be given elsewhere; see also [27] for similar calculations.

[35] A. Golubov, M. Kupriyanov, and E. Il'ichev, Rev. Mod. Phys. 76, 411 (2004).

[36] F. Konschelle, J. Cayssol, and A. I. Buzdin, Phys. Rev. B 78, 134505 (2008).

[37] J.-D. Pillet, C. H. L. Quay, P. Morfin, C. Bena, A. L. Yeyati, and P. Joyez, Nat. Phys. 6, 965 (2010).

[38] G. E. Blonder, M. Tinkham, and T. M. Klapwijk, Phys. Rev. B 25, 4515 (1982).

[39] P. F. Bagwell, Phys. Rev. B 46, 12573 (1992). 